\title{
Decontamination performance of air filter paper impregnated with zirconium hydroxide on sulfur mustard
}

\author{
Xingqi Huang ${ }^{1}$, Ting Zhao ${ }^{2}$, Chunxiao Yan ${ }^{1, a}$, Yanren $\mathrm{Jin}^{2}$, Yue $\mathrm{Wu}^{2}$, Lingxuan Zhang ${ }^{2}$ \\ ${ }^{1}$ Institute of NBC Defense, Chinese PLA Army, Beijing, China \\ ${ }^{2}$ Shanxi Xinhua Chemical Defense Equipment Research Institute Co., Ltd. Shanxi, China
}

\begin{abstract}
Using zirconium hydroxide as a decontaminant, a kind of self-decontaminating air filter paper that can effectively degrade HD was successfully prepared by impregnating. The morphology and filtration efficiency of the filter paper before and after immersing were compared. The filtration efficiency increased linearly and slowly, with the regression equation: $\eta=0.0001 L+99.971$. The liquid-solid decontamination reaction of HD on zirconium hydroxide powder and self-decontaminating filter paper conformed to the kinetic of quasi-first-order reaction and found that half-lives were $0.4 \mathrm{~h}$ and $2.1 \mathrm{~h}$ respectively. Good degradation performance for HD was exhibited and the degradation rate reached more than $99 \%$ in 12 hours. Reaction products were analysed by GC-MS, which displayed that HD was decomposed through the substitution of chlorine and the elimination of $\mathrm{H}$ on $\alpha$-C. The ultimate decontamination products were thiodiglycol and 2hydroxyethyl vinyl sulfide without erosive toxicity.
\end{abstract}

\section{Introduction}

Toxic aerosol is blocked through a filter layer made of fibre material in the gas mask, so as to avoid injury to personnel. However, the filter paper, having no decontamination effect, can only filter poisonous smoke and mist. The sublimation of poisonous smoke and the evaporation of poisonous mist deposited on the filter paper may threaten its use as well as the safety of human and environment after missions, and also increase the difficulty of its scrap disposal ${ }^{[1]}$. Therefore, it is of vital necessity for the modification of filter paper to make it have the function of self-decontamination.

Sulfur mustard (2,2'-dichloroethyl sulfide), also known as HD, was invented at the end of the 19th century and was first used by Germany in the Battle of Ypres in Belgium during World War I, leading to a large number of casualties among forces ${ }^{[2]}$. It is a blister agent with highly erosive poison, which can cause tissue cell necrosis until ulceration, and has no specific drugs. It is called "the king of chemical warfare agents" and can be produced conveniently and inexpensively ${ }^{[3,4]}$. Despite the restrictions of the international convention, HD continues to threaten civilians and military personnel due to legacy historical issues, such as the Qiqihar poisoning incident, and the continued production, stockpiling and development of HD [5]. Decontaminants that can effectively decompose HD through oxidation, hydrolysis, and dehydrohalogenation have been investigated by relevant scholars ${ }^{[6]}$. However, there are some remaining problems: (1) some degradation products are still very toxic; (2) decontaminants are corrosive, not environmentally friendly; and (3) their activity will decrease over time, which may pose a huge risk to mankind ${ }^{[7]}$. Therefore, it is necessary to continuously develop new materials or methods for decontamination of HD.

Zirconium hydroxide $\left(\mathrm{Zr}(\mathrm{OH})_{4}\right)$, one of the metal hydroxide materials that have both acid-base and redox properties, is an amorphous porous material with multiple active centres such as hydroxyl groups, coordinated unsaturated metal cations $\left(\mathrm{Zr}^{\delta^{+}}\right)$and oxygen vacancies distributed on the surface ${ }^{[8]}$. The ability of zirconium hydroxide to degrade HD was evaluated and the half-life monitored by ${ }^{13} \mathrm{C}$ NMR spectra was $2.3 \mathrm{~h}{ }^{[9]}$, revealing an excellent decontamination function on HD. Simultaneously, taking into account it without corrosivity, it is feasible to immerse $\mathrm{Zr}(\mathrm{OH})_{4}$ into filter paper. The preparation of improved filter paper with decontaminating HD can not only provide crucial materials for the design of better gas masks, but also solve the problem of secondary poisoning caused by poison aerosols, improving the protective performance of gas masks and ensuring the safety of protective equipment.

\section{Materials and Methods}

\subsection{Chemicals}

Filter paper was obtained from Tianlun Papermaking Technology Development Centre (Tianjin, China). Zirconium hydroxide $\left(\mathrm{Zr}(\mathrm{OH})_{4}\right.$, AR grade) was purchased from Tianjin Damao Chemical Machinery Factory. Petroleum ether (PE, boiling temperature range $90 \sim 120^{\circ} \mathrm{C}$, AR grade) and acetonitrile (AR grade) were

\footnotetext{
a Corresponding author: 972205784@fhhy2.onexmail.com
} 
obtained from Bafang Agents Inc. (Beijing, China), as extractants.

HD (of more than 98\% purity) was supplied by synthetic chemistry division of our establishment. Due to their high toxicity and dangerousness, they were handled only by well-trained personnel using appropriate procedures carefully in very small quantities.

The blue reagent ( $\mathrm{T}-135$ reagent) was made in the laboratory by dissolving thymol phthalein $(2 \mathrm{~g})$ and potassium hydroxide (KOH, $0.56 \mathrm{~g}$, AR grade) in $340 \mathrm{~mL}$ of anhydrous ethanol, and dilute to $400 \mathrm{~mL}$ with $\mathrm{H}_{2} \mathrm{O}$.

\subsection{Preparation of self-decontaminating filter paper}

Filter paper was cut to obtain a circular sample with a diameter of $6 \mathrm{~cm}$. Zirconium hydroxide powder was dried at $60^{\circ} \mathrm{C}$ for $2 \mathrm{~h}$ and sieved to 200 mesh after grinding. A certain amount of powder was weighed and placed in a petri dish for $1.5 \mathrm{~h}$ of ultrasound. The cutting paper was immersed in the suspension for $3 \mathrm{~min}$ and placed on the gauze net. The impregnated paper was placed in a blast drying box at $35^{\circ} \mathrm{C}$ for $6 \mathrm{~h}$ to obtain self-decontaminating filter paper.

Weighing the filter paper before and after immersing to calculate the retention rate. The formula is as follows. Where $L$ is the retention rate of zirconium hydroxide, \%. $m_{1}$ is the weight of filter paper before immersing, g. $m_{2}$ is the weight of self-decontaminating filter paper, $g$.

$$
L=\left(m_{2}-m_{1}\right) / m_{1} \times 100 \%
$$

\subsection{Characterization and performance test}

Scanning electron microscope (SEM, Regulus 8100, Hitachi Inc., Japan) was used to observe the morphological changes of filter paper. The filtration efficiency was tested by oil mist method. The oil mist generated by the spray generator was passed through the filter paper, and the concentration before and after the permeation were measured with a turbidimeter. The oil mist concentration is $2000 \sim 2500 \mathrm{mg} / \mathrm{m}^{3}$ and the airflow specific velocity is $0.32 \mathrm{~L} / \mathrm{min} / \mathrm{cm}^{2}$. Permeability coefficient $\left(K_{f}, \%\right)$ was calculated as formula (2) and filtration efficiency $(\eta, \%)$ was calculated as formula (3).

$$
\begin{gathered}
K_{f}=\frac{C_{t}}{C_{0}} \times 100 \% \\
\eta=1-K_{f}
\end{gathered}
$$

Where, $K_{f}$ is the transmittance of filter paper, \%. $C_{0}$ and $C_{t}$ are the concentration of oil mist before and after capturing respectively, $\mathrm{mg} / \mathrm{m}^{3} . \eta$ is the filtration efficiency of filter paper, $\%$.

\subsection{Decontamination of HD}

\subsubsection{The calibration curve of the HD standard solution}

Alkalized thymol phthalein (T-135 reagent), reacting with
HD to become a stable orange-yellow substance after acidification, is used as an indicator to measure the concentration of HD. The absorbance was measured by an ultraviolet-visible spectrophotometer $\left(\lambda_{\max }=447 \mathrm{~nm}\right)$. The calibration curve was obtained by plotting the assayed quantity (y-axis) versus the concentration (x-axis) of the HD solution.

\subsubsection{Decontamination of HD by zirconium hydroxide powder}

Add $1 \mathrm{~g}$ of $\mathrm{Zr}(\mathrm{OH})_{4}$ powder to $5 \mu \mathrm{L}$ of HD containing on the bottom of the conical flask, shake it evenly and seal the container at a constant temperature of $25 \pm 1{ }^{\circ} \mathrm{C}$ for different time. $15 \mathrm{~mL}$ of petroleum ether, as extractant, was added into the container and shook for $20 \mathrm{~s}$ continuously. After $15 \mathrm{~min}$ of sample resting, petroleum ether phase was sampled for residual HD determination and the recovery calculation. According to the standard curve, the concentration of residual HD can be obtained. The formula of decontamination rate is as follows.

$$
D=\left(1-\frac{C_{t}}{C_{0}}\right) \times 100 \%
$$

Where $D$ is the decontamination rate of $\mathrm{HD}, \% . C_{t}$ is the detected concentration of residual $\mathrm{HD}, \mathrm{mg} / \mathrm{L} . C_{0}$ is the initial concentration of $\mathrm{HD}, \mathrm{mg} / \mathrm{L}$.

\subsubsection{Decontamination of HD by self- decontaminating filter paper}

$5 \mu \mathrm{L}$ of HD was transferred on the well-prepared selfdecontaminating filter paper, positioned in the conical flask, with a micropipette. Sealing with parafilm, the container was placed in a thermostat with the temperature of $25 \pm 1^{\circ} \mathrm{C}$, and the analysis method for the residual HD was the same as above.

\subsection{Detection of reaction products}

The same quantity of HD and decontaminant was mixed as the reaction system, using acetonitrile instead of petroleum ether as the extractant. Reaction products were analyzed by gas chromatography-mass spectrometry (7890A GC system coupled with 5975C MSD detector) of Agilent Technologies equipped with an HP-5MS capillary column $(30 \mathrm{~m} \times 0.25 \mathrm{~mm} \times 0.25 \mu \mathrm{m})$. The measurement conditions were as follows: carrier gas is high-purity helium with a flow rate of $50 \mathrm{~mL} / \mathrm{min}$, injection port temperature is $250^{\circ} \mathrm{C}$, detector temperature is $150^{\circ} \mathrm{C}$, ion source temperature is $230^{\circ} \mathrm{C}$, temperature programming is $50{ }^{\circ} \mathrm{C}$ for 1 minute, then $15^{\circ} \mathrm{C} / \mathrm{min}$ to $250{ }^{\circ} \mathrm{C}$ for 2 minutes. The shunt ratio is $50: 1$ and the ionization voltage is $70 \mathrm{eV}$.

\section{Results and Discussion}

\subsection{Characterization and performance test of self- decontaminating filter paper}




\subsubsection{SEM characterization}

The SEM images of the filter paper before and after immersion are displayed in Figure 1, showing the result of 2000 times magnification of the morphology and structure. Before impregnation, the porous structure is formed by glass fiber as the skeleton. Particulate solids appear between the fibers of the filter paper after impregnation and the surface is uneven, indicating that the zirconium hydroxide is loaded on the filter paper and may has an impact on the internal structure of the paper.
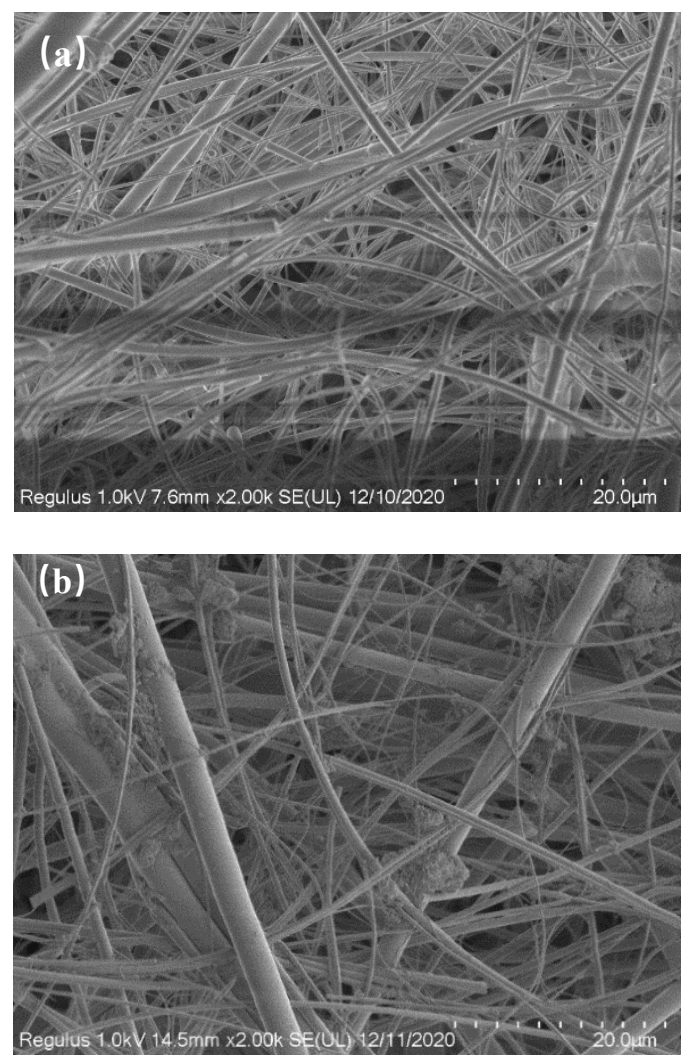

Figure 1. Scanning electron microscopy (SEM) images of filter paper before(a) and after(b) immersion

\subsubsection{Test for filtration efficiency}

Filtration efficiency of the air filter paper with different retention rate of $\mathrm{Zr}(\mathrm{OH})_{4}$ is shown in Figure 2. The filtration efficiency increased after immersion relative to the previous numerical value of $99.975 \%$. With the increase of the retention rate of decontaminant, it showed a linear growth trend, and the regression equation is $\eta=0.0001 L+99.971$. The $\mathrm{Zr}(\mathrm{OH})_{4}$ impregnated into the paper blocked the fibre pores and caused the diameter of fibre pores to decrease. As a consequence, the amount of oil mist deposited on the filter paper accelerated and the filtration efficiency increased.

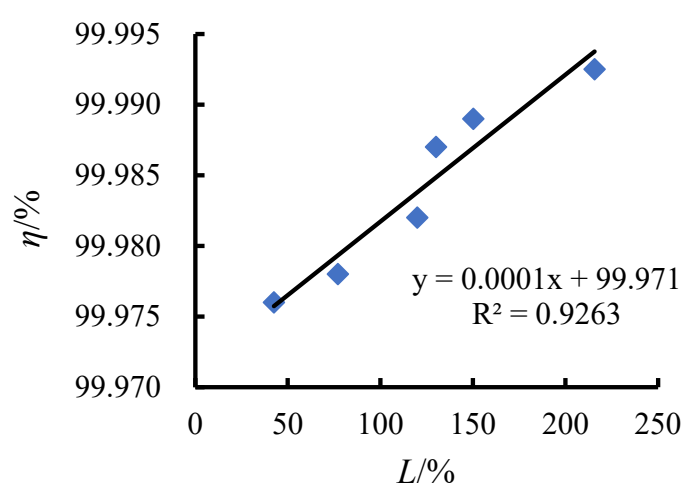

Figure 2. Filtration efficiency of self-decontaminating filter paper varies with the retention rate of $\mathrm{Zr}(\mathrm{OH})_{4}$

\subsection{Decontamination of HD}

The concentration of HD (y, $\mu \mathrm{g} / \mathrm{ml})$ versus absorbance ( $\mathrm{x}$, Abs.) showed a good linear relationship in the range of $0.12 \sim 0.7$ and a linear regression equation $y=151.55 x$ was obtained with a linear correlation coefficient $\mathrm{R}^{2}=0.9995$.

\subsubsection{Degradation of $H D$ on zirconium hydroxide powder}

The residual HD concentration was determined at various intervals of time and kinetic plots were made by taking the concentration on Y-axis and time on X-axis. A smooth curve manifesting the degrading process of $\mathrm{HD}$ on $\mathrm{Zr}(\mathrm{OH})_{4}$ powder was illustrated in Figure 3. The longer the time, the better the decontamination effect, and the degradation of HD was fast initially and then slowed down to achieve a steady state. According to the quasi-first-order reaction rate equation (formula (5)), the calculated degradation rate constant $(k)$ and half-life $\left(t_{1 / 2}\right)$ are 1.372 $\mathrm{h}^{-1}$ and $0.4 \mathrm{~h}$. The degradation rate can reach more than $99 \%$ after 4 hours.

$$
\operatorname{Ln}\left(C_{t} / C_{0}\right)=k t+b
$$

Where $C_{t}$ is the detected concentration of residual HD, $\mathrm{mg} / \mathrm{L} . C_{0}$ is the initial concentration of HD, $\mathrm{mg} / \mathrm{L} . k$ is the degradation rate constant, $\mathrm{h}^{-1} . t$ is the reaction time and $b$ is a constant.

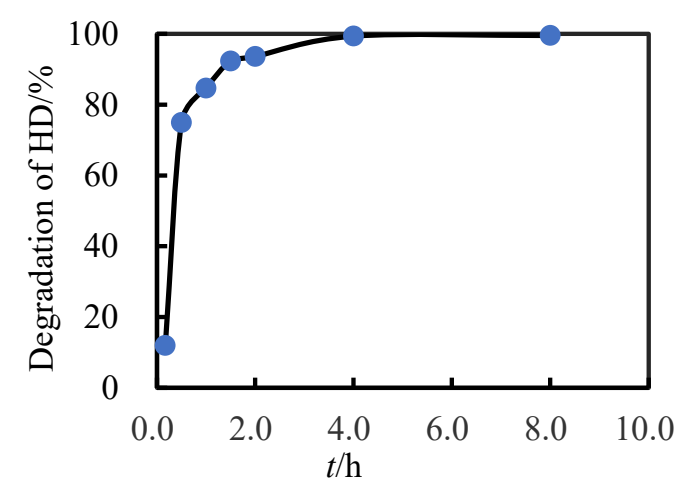

Figure 3. Kinetic of degradation of HD on zirconium hydroxide powder 


\subsubsection{Degradation of HD on self-decontaminating filter paper}

The kinetic curve of degradation of HD on selfdecontaminating filter paper with the retention rate of $\mathrm{Zr}(\mathrm{OH})_{4}$ of about $120 \mathrm{wt} \%$ and the degradation rate of HD on self-decontaminating filter paper with different retention rate of $\mathrm{Zr}(\mathrm{OH})_{4}$ for $24 \mathrm{~h}$ were displayed in Figure 4 and Figure 5 respectively.

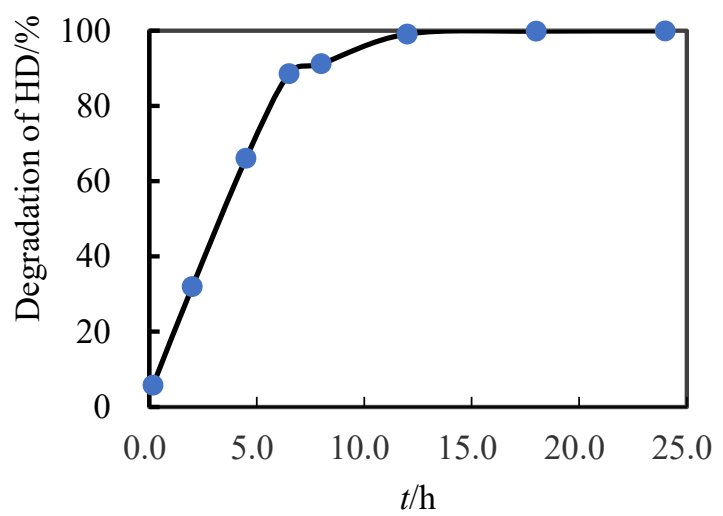

Figure 4. Kinetic of degradation of HD on selfdecontaminating filter paper

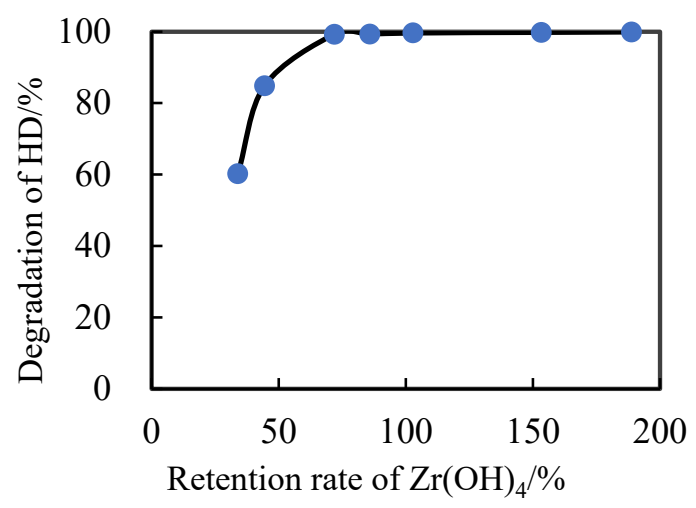

Figure 5. Degradation of HD on self-decontaminating filter paper varies with the retention rate of $\mathrm{Zr}(\mathrm{OH})_{4}$

It can be seen from Figure 4 that the degradation of HD presented a trend of rapid first and then slowed down, indicating that the degradation reaction is a first-order reaction. The initial response is fast because the HD droplets spread quickly on the filter paper and fully contact the surface of the decontaminant. When the $\mathrm{Zr}(\mathrm{OH}) 4$ on the surface of paper is consumed, further reactions occur inside the paper and HD needs to penetrate into the interior to continue the degradation process. The liquid penetration rate inside the paper being slow as well as insufficient amount of internal $\mathrm{Zr}(\mathrm{OH}) 4$ within the reach of HD droplets caused the reaction speed to slow down. Rate constant value $(\mathrm{k})$ and half-lives (t1/2) are $0.329 \mathrm{~h}-1$ and $2.1 \mathrm{~h}$, which are calculated by first-order rate equation.

It can be seen from Figure 5 that as the retention rate of $\mathrm{Zr}(\mathrm{OH}) 4$ increases, the degradation rate of HD shows an increasing trend. When the retention rate reaches more than $70 \%$, the degradation rate for $24 \mathrm{~h}$ of decontamination can reach more than $99 \%$. According to the conclusions obtained in Figure 2, it can be seen that the filtration efficiency at this retention rate is $99.979 \%$.

\subsection{Reaction products and mechanism}

In order to study the mechanism of degradation of HD on self-decontaminating filter paper, reaction mixtures were analyzed by GC-MS and the data were verified for product fragmentation patterns based on NIST Mass Spectral Database. 2-chloroethyl vinyl sulfide (with $\mathrm{m} / \mathrm{z}$ values at 45,60,73,122), 2-hydroxyethyl vinyl sulfide (with $\mathrm{m} / \mathrm{z}$ values at $45,60,73,104)$, 2-hydroxyethyl-2'-chloroethyl sulfide (with $\mathrm{m} / \mathrm{z}$ values at $45,61,73,91,109,140$ ) and thiodiglycol (with $\mathrm{m} / \mathrm{z}$ values at18,28,61,104) were detected (Figure 6).

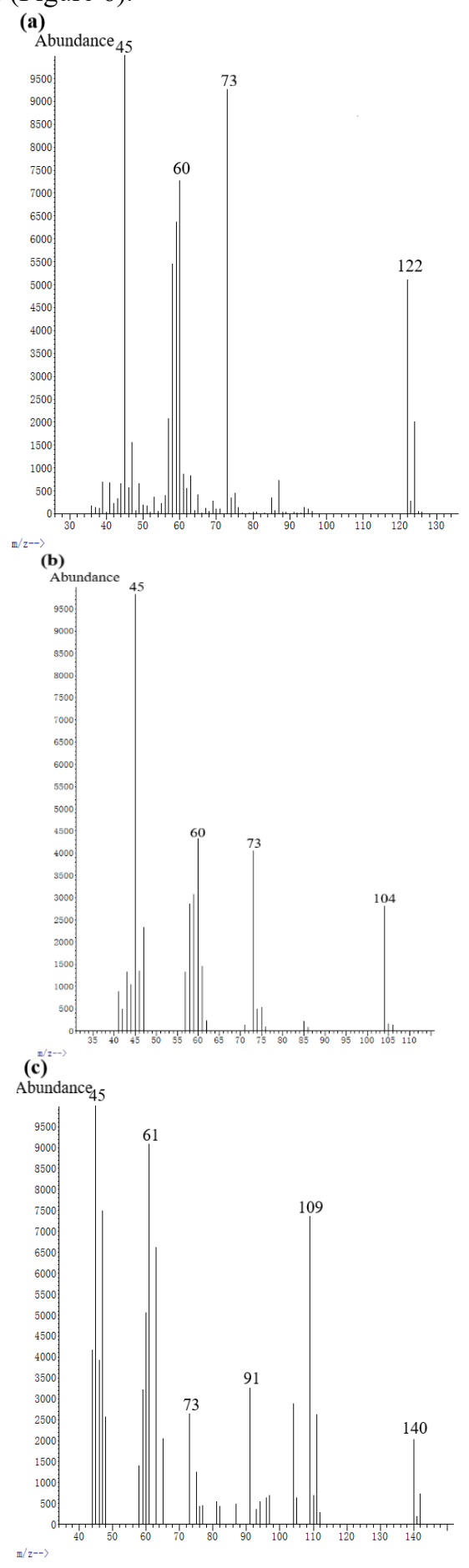




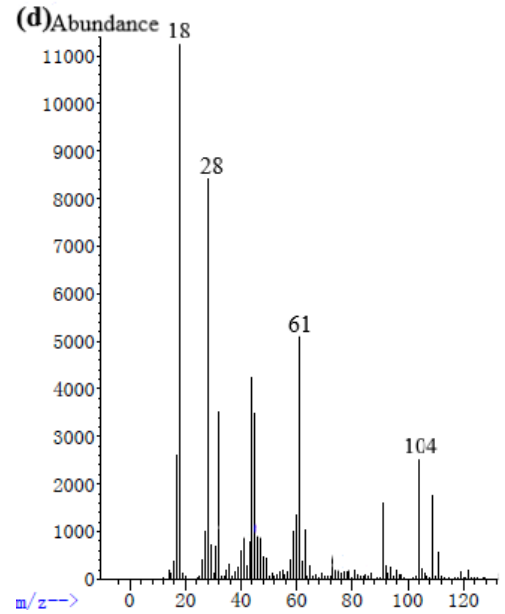

Figure 6. Mass spectra of the products extracted from HD after being degraded by $\mathrm{Zr}(\mathrm{OH})_{4}$ : (a) 2-chloroethyl vinyl sulfide; (b) 2-hydroxyethyl vinyl sulfide ;(c) 2-hydroxyethyl-2'-chloroethyl sulfide; (d) thiodiglycol

The inferred reaction mechanism is shown in the scheme 1. Reaction pathway (1): $\mathrm{H}$ on $\alpha-\mathrm{C}$ was eliminated followed by hydrolysis. Reaction pathway (2): the first step is the attack of sulfur atoms to form sulfide ions, and then water attacks the carbon atoms on the ring to open the ring to form half mustard gas and $\mathrm{HCl}$. Half mustard gas is still erosive, and it interacts with water. The reaction continues to produce thiodiglycol and $\mathrm{HCl}$.

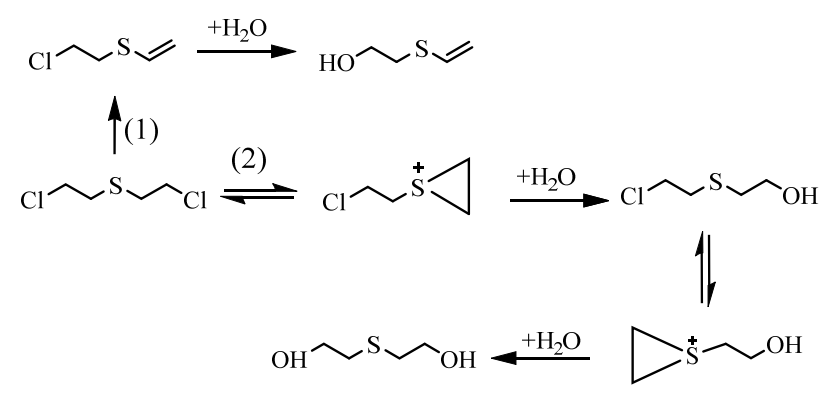

Scheme 1. Reaction pathways of HD on self-decontaminating filter paper

\section{Conclusions}

The self-decontaminating air filter paper with fine filtration efficiency was successfully prepared using zirconium hydroxide as the decontaminant. Kinetics of degradation of HD on zirconium hydroxide powder and self-decontaminating filter paper were studied by the chemical indicator method and found that half-lives are $0.4 \mathrm{~h}$ and $2.1 \mathrm{~h}$ respectively. When the retention rate of $\mathrm{Zr}(\mathrm{OH})_{4}$ reached more than $70 \%$, the filter paper can completely degrade HD in $24 \mathrm{~h}$. At this retention rate, the filtration efficiency is $99.979 \%$. GC-MS results revealed that HD can be efficiently degraded by the selfdecontaminating filter paper through hydrolysis and elimination reactions and ultimate products are thiodiglycol and 2-chloroethyl vinyl sulfide, which are non-erosive.

\section{References}

1. Y.Q. Li, Q. Gao, Y.S. Zhou, L.J. Zhang, Y.X. Zhong, Y. Ying, M.C. Zhang, Y.Q. Liu, Y.A. Wang. J. Hazard. Mater.358:113-121. (2018)

2. G.J. Fitzgerald. Am. J. Public Health. 98: 611-625. (2008)

3. M.J. Geraci. Ann Pharmacother. 42:237-246. (2008)

4. K. Ghabili, P.S. Agutter, M. Ghanei, K. Ansarin, Y. Panahi, M.M. Shoja. Crit. Rev. Toxicol. 41: 384-403. (2011)

5. Y. Liu, C.T. Buru, A.J. Howarth, J.J. Mahle, J.H. Buchanan, J.B. DeCoste, J.T. Hupp, O.K. Farha. J. Mater. Chem. A Mater. 4:13809-13813. (2016)

6. A.J. Howarth, C.T. Buru, Y. Liu, A.M. Ploskonka, K.J. Hartlieb, M. McEntee, J.J. Mahle, J.H. Buchanan, E.M. Durke, S.S. Al-Juaid. Eur.Chem.23: 214-218. (2017)

7. H. Wang, G.W. Wagner, A.X. Lu, D.L. Nguyen, J.H. Buchanan, P.M. McNutt, C.J. Karwacki. ACS Appl. Mater. Interfaces. 10: 18771-18777. (2018)

8. X.B. Wang, L. Li, K. Li. Chin. J. Environ. Eng.14: 2761-2773. (2020)

9. T.J. Bandosz, M. Laskoski, J. Mahle, G. Mogilevsky, G.W. Peterson, J.A. Rossin, G.W. Wagner. J. Phys. Chem. C. 116: 11606-11614. (2012) 